\title{
Emotion processing in infancy: specificity in risk for social anxiety and associations with two year outcomes
}

Article

Accepted Version

Creswell, C., Cooper, P., Giannakakis, A., Schofield, E., Woolgar, M. and Murray, L. (2011) Emotion processing in infancy: specificity in risk for social anxiety and associations with two year outcomes. Journal of Experimental

Psychopathology, 2 (4). pp. 490-508. ISSN 2043-8087 doi: https://doi.org/10.5127/jep.018911 Available at https://centaur.reading.ac.uk/25347/

It is advisable to refer to the publisher's version if you intend to cite from the work. See Guidance on citing.

To link to this article DOI: http://dx.doi.org/10.5127/jep.018911

Publisher: Textrum

All outputs in CentAUR are protected by Intellectual Property Rights law, including copyright law. Copyright and IPR is retained by the creators or other copyright holders. Terms and conditions for use of this material are defined in the End User Agreement. 


\section{CentAUR}

Central Archive at the University of Reading

Reading's research outputs online 


Journal of
TEXTRUM

\section{Emotion processing in infancy: Specificity in risk for social 2 anxiety and associations with two-year outcomes}

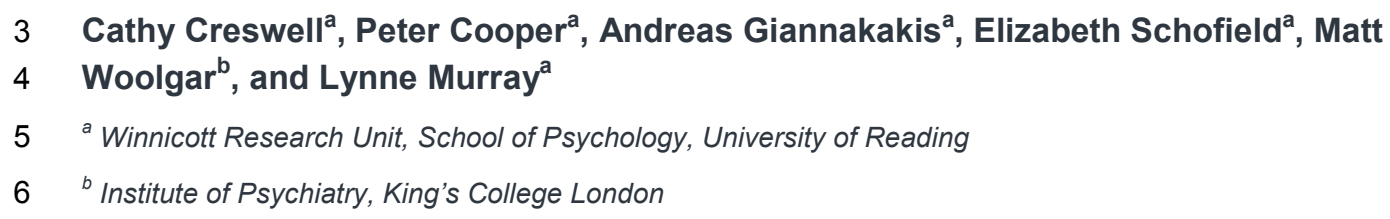

\section{Abstract}

8 The current study examined the specificity of patterns of responding to high and low intensity negative 9 emotional expressions to infants of mothers with social phobia, and their association with child outcomes 10 at two years of age. Infants of mothers with social phobia, generalised anxiety disorder (GAD) or no 11 history of anxiety were shown pairs of angry and fearful emotional expressions at 10 weeks of age. 12 Symptoms of social withdrawal, anxiety and sleep problems were assessed at two years of age. Only 13 infants of mothers with social phobia showed a tendency to look away from high intensity fear faces; however infants of mothers with both social phobia and GAD showed a bias towards high intensity angry faces. Among the offspring of mothers with social phobia, anxiety symptoms at two years of age were associated with a preference for high intensity fear faces in infancy. The reverse pattern was found amongst the offspring of non-anxious mothers. These findings suggest a possible specific response to emotional expressions among the children of mothers with social phobia.

(c) Copyright 2011 Textrum Ltd. All rights reserved.

Keywords: mother, infant, anxiety, information-processing, emotional expression, looking times

Correspondence to: Cathy Creswell, Winnicott Research Unit School of Psychology, University of Reading, Whiteknights, Reading, Berkshire RG6 6AH. Email: c.creswell@reading.ac.uk

1. Winnicott Research Unit School of Psychology, University of Reading, Whiteknights, Reading, Berkshire RG6 $6 \mathrm{AH}$

\section{Institute of Psychiatry, King's College London}


 \\ Table of Contents}

29 Introduction

References

\section{Introduction}

Social phobia is a chronic, disabling disorder, characterised by intense fear of negative evaluation by others, that affects between 7 and $13 \%$ of Western populations (Furmark, 2002). Adults and children with high levels of social anxiety have been shown to evidence distinctive patterns of responding to negative emotional faces (e.g. Bögels \& Mansell, 2004; Garner, 2010). It remains unclear, however, whether responses to social emotional information have a specific role in the aetiology of social anxiety. Tentative support comes from our previous report of responses to emotional faces amongst 10 week old infants of mothers with social phobia. We found that, whereas 10 week old infants of non-anxious mothers showed increased initial orientation and overall looking to high, versus low, fear faces, infants of mothers with social phobia showed the reverse pattern (Creswell et al., 2008). The purpose of this study is to extend these findings to address the following questions: (i) is this pattern of responding to emotional faces specific to infant offspring of mothers with social phobia, in comparison to offspring of mothers with another anxiety disorder (here, Generalised Anxiety Disorder (GAD)), and (ii) is infant response style to emotional faces associated with later anxiety symptoms, assessed here at 2 years of age.

\section{Responses to emotional expressions: role in the development of anxiety}

A number of studies have demonstrated that socially anxious individuals show distinctive attentional responses to emotional faces (e.g. Bögels \& Mansell, 2004), which appear to reflect a heightened sensitivity to potentially threatening faces at early stages of processing. For example, compared to nonanxious controls, socially anxious adults have been found to identify angry faces at lower levels of intensity (Joorman \& Gotlib, 2006). There is increasing support for the idea that this initial sensitivity, or hypervigilance, is followed by avoidance, which is viewed as an attempt to reduce discomfort associated with the stressful stimuli (e.g. Mogg, Bradley, de Bono, \& Painter, 1997). In turn, this avoidance leads to heightened potentiation of the fear response and reduced habituation, thereby maintaining the fear response (Mogg \& Bradley, 1998).

There is a well established familial basis to the development of social phobia (e.g. Lieb et al., 2000; Mancini, van Ameringen, Szatmari, Fugere, \& Boyle, 2006); from a meta-analysis of twin studies a heritability estimate of .65 was estimated for social anxiety (Beatty, Heisel, Hall, Levine, \& La France, 2002), although many studies suggest a more modest effect (e.g. 0.31 - Kendlar et al., 1992). It is 
important to note, however, that most studies have suggested that much of the inherited component is common across anxiety and depressive disorders (Andrews, 1996; Eley, 1999), although Kendlar, Myers, Prescott, and Neale (2001) concluded that $13 \%$ of the variance in social anxiety was accounted for by specific genetic factors. The phenotypic expression of this genetic risk is of much interest, and candidates that have been considered have focused on temperamental characteristics, such as behavioural inhibition (Bl; Elizabeth et al., 2006), neuroticism, introversion (Bienvenu, Hettema, Neale, Prescott, \& Kendlar, 2007) and harm avoidance (Lochner et al., 2007). Theories of the role of social information processing, have also suggested that individual cognitive biases may be an integral part of, and a risk factor for, social anxiety (Richards, French, Nash, Hadwin, \& Donnelly, 2007); this proposition has been labelled the 'integral hypothesis', and can be contrasted with two competing hypotheses, namely, the 'inferred bias hypothesis' (McClure \& Nowicki, 2001; Richards et al., 2007) and the 'inhibition hypothesis' (Kindt \& van den Hout, 2001). The 'inferred bias hypothesis' suggests that social anxiety may itself interfere with social information processing, leading to the expectation that social information processing biases should increase with age, and are unlikely to be apparent in early infancy. The 'inhibition hypothesis' suggests, by contrast, that a bias for threatening stimuli is normal in young children, but becomes inhibited in non-anxious children through development. Support for the 'integral hypothesis' comes from two studies to date. First, Joorman, Talbot, and Gotlib (2007) used a dot-probe paradigm and presented sad-neutral and happy-neutral face pairs to 21 daughters (aged 9 to 14 years) of mothers with a history of depression (the 'high risk' group) and 20 daughters of mothers with no history of an Axis I disorder (the 'low risk' group). High, but not low, risk daughters selectively attended to sad faces, whereas the reverse pattern was found for happy faces. While these findings may have been a result of environmental influence, it is notable that they were not attributable to current symptoms of depression (or anxiety), and, as such, go against the 'inferred bias' hypothesis. In relation to anxiety and threat faces, we reported preliminary support for the 'integral bias hypothesis' from a study of looking responses of infant offspring of mothers with social phobia and non-anxious mothers assessed at 10 and 16 weeks and 10 months of age. We were interested in infants' responses to high versus low intensity emotional faces, based on findings with adults that suggest that high levels of social anxiety are associated with avoidance of extreme positive and negative (but not neutral) emotional expressions, under conditions of socially-evaluative threat (Mansell, Clark, Ehlers, \& Chen, 1999). Infants were presented with a series of pairs of morphed faces, in which one face represented $50 \%$ emotional expression (of either happiness, fear or anger) while the other represented a high $(75 \%$ or $100 \%)$ or low $(0 \%$ or $25 \%)$ intensity version. Although there was no direct examination of the impact of social threat, all conditions involved the infant being confronted with emotional faces facing directly towards them. Infants of non-anxious mothers, at 10 weeks and 10 months, were more likely to initially orient to high (vs. low) intensity fear faces, when presented to the right of a control $(50 \%)$ expression and they showed increased overall looking to the high intensity fear face. In contrast, 10 week and 10 month infants of mothers with social phobia were more likely to look initially to the low intensity fear faces and show increased overall looking to the low intensity fear face (Creswell et al., 2008). Thus, while we found no evidence for initial hypervigilance to threat faces, the results were consistent with a tendency for infants at genetic risk of social phobia to avoid high intensity emotional expressions. Conclusions must remain tentative however, as although the infants were very young, at 10 weeks they have still had substantial social experience (although it was notable that responses to fear faces were not associated with measures of maternal social behaviour). In addition, it could not be established whether infant responses to emotional expressions are specifically associated with maternal social phobia, as opposed to other maternal anxiety disorders. Furthermore, since no longer-term outcomes were considered, it could not be established whether infant responses to emotional faces are a precursor to longer term anxiety symptoms. 


\section{Responses to emotional expressions: specificity to social anxiety}

While evidence from non-clinical populations suggests that there is an emotion-congruent effect, in which particular patterns of hypervigilance- avoidance to emotional stimuli are associated with anxious versus depressive symptoms (e.g. Bradley, Mogg, \& Lee, 1997; Bradley, Mogg, Falla, \& Hamilton, 1998), it is less clear whether there is such specificity between anxiety subtypes. For example, a lack of specificity is suggested by findings that hypervigilance to threat related faces (anger and/or fear) is associated with the presence of both social phobia (e.g. Mogg, Bradley, Miles, \& Dixon, 2004) and GAD (e.g. Bradley, Mogg, White, Groom, \& de Bono, 1999). However, support for disorder-specific processing of emotional material in social anxiety comes from evidence that both hypervigilance (Mogg \& Bradley, 2002) and subsequent avoidance (e.g. Garner, Mogg, \& Bradley, 2006) are significantly associated with social anxiety after controlling for trait anxiety (Bögels \& Mansell, 2004).

Few studies have examined associations between responses to emotional expressions and social anxiety in children. Those that have done so provide support for differential attentional responding among highly anxious vs. non-anxious children (all to date using dot-probe methodologies); however, the issue of anxiety subtype specificity is unresolved. Stirling, Eley, and Clark (2006) presented 79 children (8-11 years) from a community population with negative (fearful and angry), neutral and positive faces within a dot-probe paradigm. In this study, stimuli were presented for a relatively long duration (1000 $\mathrm{ms})$, preventing conclusions being drawn about rapid attentional responses to emotional stimuli; nevertheless, over this longer period, avoidance of negative facial expressions was found to be associated with child self-reported social anxiety, but not general anxiety- i.e. findings consistent with an emotion-congruent account. In contrast, a number of other studies have failed to find such evidence. Thus, Monk et al. (2006) presented adolescents with GAD (age 9-17 years) with angry/neutral face pairs, again for a relatively long duration $(500 \mathrm{~ms})$ and also found that the clinical group showed avoidance of negative (angry) faces. Furthermore, in a subsequent study in which stimuli were presented over a shorter duration $(17 \mathrm{~ms})$, adolescents with GAD showed an initial attentional bias towards angry faces (Monk et al., 2008). Hypervigilance for angry faces was also found among more severe, but not milder, cases of GAD in children aged 7 to 12 years (Waters, Mogg, Bradley, \& Pine, 2008). In a study of 101 children with mixed anxiety disorders and 51 non-anxious control children, Roy et al. (2008) reported that while the clinical group showed a bias towards angry (but not happy) faces, there were no significant associations with specific anxiety diagnoses. In sum, although overall conclusions that can be drawn are limited by the different age-ranges of participants, and differences in experimental methods (e.g. different lengths of time for the presentation of stimuli, and inclusion of different types of emotional expressions), the balance of evidence provides only limited support for specificity of responses to emotional expressions amongst children according to particular anxiety subtypes.

The current study aimed to extend our previous research, first, by determining whether the biases found in responding to emotional expressions were specific to those offspring at risk of social phobia, by comparing them to a group of offspring with a more general risk of anxiety (i.e. offspring of mothers with GAD), as well as differing from offspring of non-anxious, control, mothers. While dot probe methods are clearly not directly applicable with infant samples, numerous studies have assessed infant attentional responses by recording patterns of infant looking to different stimuli (see Creswell et al., 2008). Specifically, because social anxiety is associated with avoidance of extreme emotional expressions, even when positive, under conditions of socially-evaluative threat (Mansell et al., 1999), we examined infant responses to extreme emotional expressions in comparison to moderate expressions. Following on from our previous report, we have focused here on responses of 10 week old infants, as it was at this age that the clearest effects of maternal social phobia were found. In our previous report we considered infant looking responses to angry, fearful and happy faces, and found significant effects only for fearful 
faces. We have accordingly followed this up with further consideration of fearful faces, but we have also continued to examine responses to angry faces, because findings of previous studies with anxious children have most consistently reported differential responding to angry faces. Finally, whereas in our previous report the index group included offspring of mothers with social phobia and comorbid GAD, as the current study is concerned with specificity, only the offspring of mothers with pure social phobia (i.e. social phobia in the absence of GAD) were included, along with a new sample of offspring of mothers with pure GAD (i.e. GAD in the absence of social phobia) were included, as well as mothers with no history of an anxiety disorder. The first hypothesis, therefore, set out to replicate our previous findings but restricting the sample to the pure social phobia group. The initial hypotheses are as follows:

1. In comparison to infants of mothers with no history of anxiety, 10 week old infants of mothers with social phobia will show reduced looking to high intensity negative emotional faces (vs. low intensity faces) when presented alongside a face of moderate intensity as indexed by looking times and initial orientation to stimuli.

2. Biases in responding to high vs. low intensity faces will be specific to infants of mothers with Social Phobia, in comparison to infants of mothers with GAD

We also aimed to examine whether infant looking responses to emotional faces were associated with later anxiety symptoms. We assessed symptoms of social and general anxiety when the children were two years of age, as behaviours indicative of strong social fears, that predict an increased incidence of social phobia in later life, have been identified in children as young as 21 months of age (Rosenbaum et al., 1992). We hypothesised as follows:

3. Responses to high/low intensity negative faces at 10 weeks will be associated with children's outcomes at 2 years of age.

The outcomes we considered were social anxiety problems (based on social withdrawal), general anxiety problems and also sleep problems. Sleep problems were included as recent findings suggest that sleep problems are an independent predictor of later anxiety, over and above early emotional/behavioural problems (Gregory \& O'Connor, 2002), and are more predictive of anxiety/depression than of other psychiatric problems (Johnson, Chilcoat, \& Breslau, 2000). Specifically, children who are reported to sleep less than others, and who show overtiredness and have trouble sleeping, have been found to be more likely to score highly on anxiety and depression 14 years later, after controlling for sex, age and anxiety/depression at earlier assessment points (Gregory, Van der Ende, Willis, \& Verhulst, 2008). Finally, we were also interested to explore whether associations between responses to emotional faces and 2 year child problems were mediated or moderated by maternal group; i.e. to establish whether responses to emotional expressions explained any association between maternal anxiety status and child symptoms at two years; and/or whether responses to emotional expressions were a better predictor of child anxiety symptoms when they occurred in the context of maternal anxiety disorder.

\section{Method}

\section{Sample}

Mothers attending routine 20-week antenatal clinics completed screening questionnaires to detect possible social phobia and generalised anxiety disorder (GAD). High scoring women were interviewed to confirm diagnosis using the Structured Clinical Interview for DSM-IV Axis I disorders (SCID-1; First, Spitzer, Gibbon, \& Williams, 1995), administered by trained clinicians. Those meeting criteria for a DSMIV diagnosis of social phobia or GAD were recruited. Controls were selected randomly from the low- 
scoring women, who were also interviewed to confirm the absence of psychiatric disorder. A total of 96 women were recruited into the social phobia group, 56 were recruited in to the GAD group, and 94 as controls (see Murray, Cooper, Creswell, Schofield, \& Sack, 2007, for full recruitment details). Due to tiredness or discomfort not all infants were able to complete the current task. Numbers providing data within the present study were 47 in the social phobia group, 43 in the GAD group, and 60 in the control group. The rate of loss for these reasons $(61 \%)$ is similar to that reported in other studies of infant looking times (e.g. 53\%; Pascalis, de Schonen, Morton, Deruelle, \& Fabre-Grenet, 1995); and the fact that it is somewhat higher is likely to reflect the relatively long duration of the current task.

\section{Procedure}

\section{7}

\section{Responses to Emotional Expressions.}

Infants were assessed in university rooms at 10 weeks of age. Infants sat on the experimenter's lap (E1), facing a grey translucent screen within a curtained-booth with low-lighting. The mother and a second experimenter (E2) sat on the other side of the curtains, out of the infant's view. Stimuli $(60 \mathrm{~cm} \times 76 \mathrm{~cm})$ were displayed in pairs about $5 \mathrm{~cm}$ apart on a grey background to match the colour of the projection screen, which was $90 \mathrm{~cm}$ distance from the infant. Stimuli were projected from behind the screen using a Sony projector.
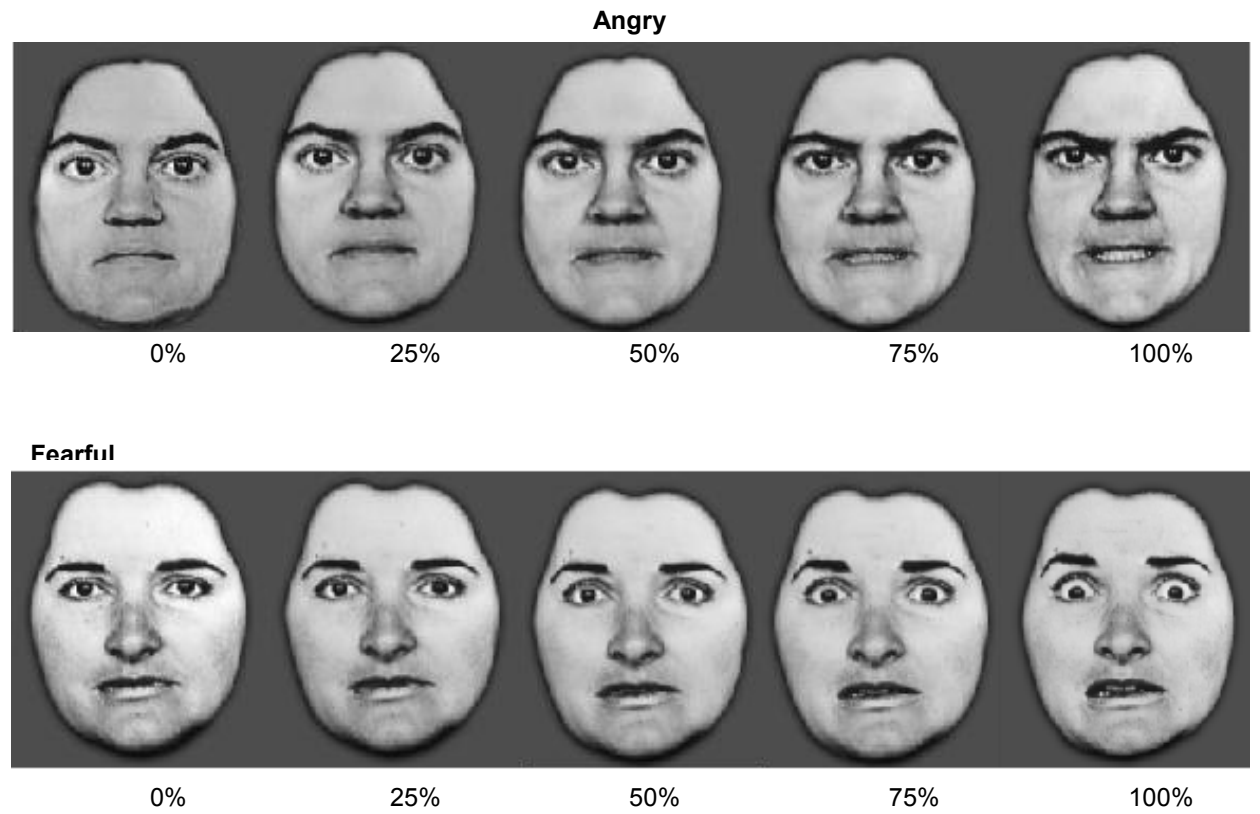

Figure 1: Emotional faces of varying intensity

Infant attention was first attracted to the screen by an image of a mobile doll. When the infant fixated on the 'attractor', experimenter E1 pressed a button to notify E2 to start the first trial. Each stimulus pair was presented for 10 seconds. Trials were continued until all stimulus pairs had been presented unless the baby became fractious or fell asleep, in which case the trial was ended prematurely (and data from that set of emotional expressions was not included in analyses). An infra-red video camera was mounted above the projection screen to film the infants' responses. A second camera was mounted behind the screen and recorded the projected images, for verification purposes. 
To assess infant responses to varying intensities of emotional expression, continua depicting different intensities of anger and fear were taken from the Facial Expressions of Emotion: Stimuli and Tests (FEEST; Young, Perrett, Calder, Sprengelmeyer, \& Ekman, 2002). Each continuum consisted of a prototype expression face (considered to be $100 \%$ intensity for that expression) from the Ekman and Friesen (1976) series, and morphed images interpolated between the prototype expression and its corresponding neutral. So, for example, the neutral face in the Ekman and Friesen series was taken to represent $0 \%$ anger, and then $25 \%, 50 \%$, and $75 \%$ angry morphs were created by interpolating between the neutral ( $0 \%$ angry) and the prototype angry (100\%) expressions. A different female model was used for each continuum; the stimuli are shown in Figure 1. The faces from the Ekman and Friesen (1976) series used to create these continua were as follows: anger (neutral NR-1-03, anger NR-2-07), fear (neutral MO-1-05, fear MO-1-23). Hairstyles and picture backgrounds were masked, so that all differences between stimuli were only emotion-relevant.

Twenty-four face pairs were shown. Within each, one face was shown at $50 \%$ intensity and the other showed varying intensities of the same emotion, either anger or fear ( 8 pairs including each emotion type; 8 pairs of happy faces were also presented but are not considered here). In this way, the standard $(50 \%)$ face could be of higher or lower emotional intensity than the comparison face. The order of emotion type was counterbalanced across participants and visits. Each face pair was presented for 10 seconds, and the same attractor was shown at the start of each trial. The duration of infant looking to each stimulus type within each trial was recorded (i.e. scorers measured duration of looks to the right face, left face, or neither face, using a button box) by scorers who observed infant behaviours on videotape and were trained to a high level of reliability on a sample of 36 videotapes (ICC = .97). From these data we extracted overall looking times to the different stimuli within each trial, which stimuli the infant looked to first, the length of the first look and the number of looks to each stimulus.

\section{Measures}

\section{Adjustment at 2 years of age.}

\section{Child Behavior Checklist (CBCL; Achenbach \& Rescorla, 2000)}

The CBCL for ages $1 \frac{1}{2}$ to 5 years was completed by mothers when their offspring were 24 months of age. The $C B C L$ is a widely used instrument composed of 100 items used to assess social and emotional adjustment in youth. The reliability and validity of this measure has been established (e.g. Achenbach, 1991). We examined the following subscales: withdrawn, DSM anxiety problems, and sleep problems. The 'withdrawn' scale consists of 8 items (e.g. 'withdrawn, doesn't get involved with others' and 'avoids eye contact'). The DSM anxiety problems scale consists of 10 items (e.g. 'Too fearful or anxious' and 'Nervous, 'high-strung', or tense'). The sleep problems scale consists of 7 items (e.g. 'trouble getting to sleep' and 'wakes up often at night') and is commonly used in sleep research (e.g. Stoleru, Nottelmann, Belmont, \& Ronsaville, 1997; Gregory \& O'Connor, 2002). Analyses were based on CBCL t-scores.

\section{Data analysis}

As is standard in infancy research (e.g. Pascalis et al., 2005), cases were excluded from analyses if the infant showed a lateral bias (i.e. they looked to one side (left or right) only throughout all trials for a particular emotion type (Angry faces: Social phobia - 2 cases, GAD - 2 cases, Control - 5 cases; Fear faces: Social phobia - 2 cases; GAD - 3 cases, Control - 3 cases)). Associations between infant responses and gender were first explored; none were significant, so gender was not considered further. In previous studies the side of stimulus presentation has been found to be important, with responses to emotional faces presented on the right side being more pronounced (e.g. Mogg \& Bradley, 1999; 2002). 
In accordance with this, we found that responses to the left and right side typically did not correlate sufficiently highly to combine them $(r=.06$ to .73$)$; therefore responses to target faces presented on the right and left were analysed separately. Participants were included if they provided data for all 8 trials within a particular emotion type, i.e. oriented to the attractor at the outset of each trial and remained sitting in front of the stimuli (even if looking away) until the final stimulus was presented. Looking responses to high and low intensity faces conformed to assumptions of parametric tests so comparisons between the pure social phobia and control groups were conducted using repeated measures analysis of variance with proportionate looking times to high and low intensity faces (i.e. time spent looking at the high intensity face vs. the $50 \%$ comparator; or time spent looking at the low intensity face vs. the $50 \%$ comparator )as the within and group as the between subjects factor. We then repeated these analyses, including the GAD group, with repeated measures ANOVA to address issues of specificity. Where significant group differences were found we, first, assessed the pattern of looking responses in more detail to explore whether differences were consistent with the hypervigilance-avoidance hypothesis using repeated measures analysis of variance for continuous looking responses (i.e. length of first look and number of looks) with looking response as the within-subjects factor and group as the between subjects factor, and chi-square test to examine associations between group and categorical looking responses (i.e. where the infant looked first).

Second, we assessed the association between looking responses and outcomes at two years. As outcome data were skewed and were not amenable to transformation, data were dichotomised, using a median split (as this represented a clear cut off point in the data), and binary logistic regression was used to investigate the main effects of group and looking times, and their interaction, in predicting 2 year outcomes. Participant numbers across trials varied due to fatigue/distress or side bias, and therefore separate analyses were conducted to avoid significant data loss.

\section{Results}

For between group comparisons, effect sizes have been reported as Pearson's correlation coefficient $r$.

Table 1: Sample characteristics

\begin{tabular}{|c|c|c|c|c|}
\hline & SP $(n=47)$ & Control $(n=60)$ & GAD $(n=43)$ & Statistic \\
\hline Child sex (\% $(n)$ male)) & $36(17)$ & $45(27)$ & $49(21)$ & $\chi^{2}(2)=1.65, p=.44$ \\
\hline $\begin{array}{l}\text { Maternal age at child birth (mean } \\
\text { years }(s d) \text { ) }\end{array}$ & $31.14(4.03)$ & $31.52(4.35)$ & $31.25(3.55)$ & $\mathrm{F}(2,145)=.13, p=.88$ \\
\hline $\begin{array}{l}\text { Marital status (\% }(n) \text { married and } \\
\text { cohabiting) }\end{array}$ & $98(46)$ & $100(60)$ & $95(41)$ & $\chi^{2}(2)=2.77, p=.25$ \\
\hline $\begin{array}{l}\text { Socio-economic status }(\%(n) \\
\text { professional/ managerial) }\end{array}$ & $70(33)$ & $72(43)$ & $74(32)$ & $\chi^{2}(2)=.43, p=.81$ \\
\hline Ethnicity $(\%(n)$ White) & $97(58)$ & $100(47)$ & $86(37)$ & $\chi^{2}(2)=9.45, p=.01$ \\
\hline
\end{tabular}

As shown in Table 1, the three study groups were similar in terms of infant sex, maternal age, socioeconomic status and marital status, and the distribution of infant gender. The groups did differ on a broad index of ethnicity, reflecting a larger number of non-white families amongst the GAD group. Nonetheless this reflected a relatively small number of non-white families in the GAD group $(n=6)$.

\section{Replication of previous findings with a pure social phobia group}

Initial analyses included only the social phobia and non-anxious control groups, in order to directly replicate the analyses conducted in our previous paper (Creswell et al., 2008), but restricting the sample to only include offspring of mothers with pure social phobia (i.e. not comorbid with GAD as in our 
previous paper). Responses to emotional expressions were first assessed based on the proportion of time spent looking at the target vs. the $50 \%$ expression. No group differences were found in the proportion of time spent looking at the target expression for fearful or angry faces, presented to either side (See Table 2). As we were particularly interested in infants' differential responses to varying intensity emotions, repeated measures analyses were conducted to compare responses to $50 \%$ morphed expressions with those to high $(75 \%$ and $100 \%)$ versus low intensity ( $0 \%$ and $25 \%)$ expressions.

Table 2: Summed proportions of infant looking time to target versus 50\% emotional expressions; Means (sds)

\begin{tabular}{lccc}
\hline Target side & SP $(\boldsymbol{n}=\mathbf{4 5})$ & Control $(\boldsymbol{n}=\mathbf{5 7})$ & GAD $(\boldsymbol{n}=\mathbf{4 1})$ \\
\hline Angry & & & \\
Low $(0 \& 25 \%)$ & & & \\
$\quad$ Right & $.71(.50)$ & $.83(.59)$ & $.86(.55)$ \\
$\quad$ Left & $1.17(.61)$ & $1.16(.59)$ & $1.00(.57)$ \\
High $(75 \& 100 \%)$ & & & \\
$\quad$ Right & $.97(.62)$ & $.72(.49)$ & $1.01(.57)$ \\
$\quad$ Left & $1.23(.66)$ & $.83(.59)$ & $1.17(.57)$ \\
Fearful & & & \\
Low $(0 \& 25 \%)$ & & & $.92(.56)$ \\
$\quad$ Right & $1.08(.68)$ & $.98(.66)$ & $1.11(.52)$ \\
$\quad$ Left & $1.14(.73)$ & $1.02(.60)$ & $.87(.56)$ \\
High $(75 \& 100 \%)$ & & & $1.03(.58)$ \\
$\quad$ Right & $.85(.58)$ & $1.00(.61)$ & $1.10(.59)$ \\
$\quad$ Left & $1.11(.61)$ & &
\end{tabular}

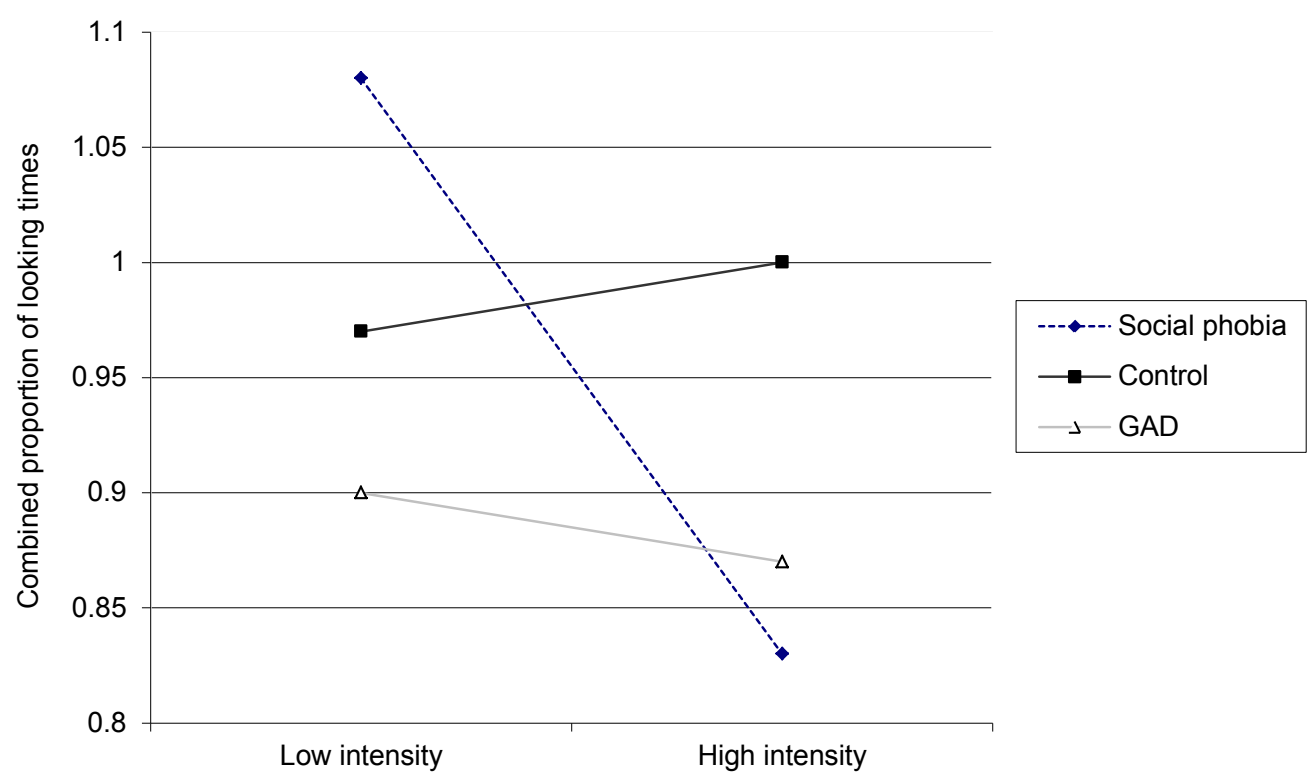

Figure 2: Interaction between participant group and level of emotional intensity of fearful expressions (presented on the right side) 
For fearful faces, the between subjects effect of group was not significant; however, the within subjects effect of intensity approached significance when the target stimulus was presented on the right, $F(1,62)$ $=3.11, p=.08, r=.22$ and there was a significant interaction effect of intensity $\times$ group, $F(1,62)=4.51$, $p=.04, r=.26$, see Figure 2. Post-hoc analyses confirmed a pattern of reduced looking to high vs. low intensity fearful faces among infants of mothers with social phobia, $t(27)=2.88, p=.01$, but not control infants, $t(35)=.25, p=.80$. This reflected a greater difference in looking to high vs. low fear faces in the social phobia group (looking to high-low fear faces: SP mean $=0.26(.47)$, control mean $=0.02(.56)$, $t(52)=2.12, p=.04)$. Neither the main effect of intensity, $F(1,57)=.14, p=.62$, nor the interaction with group, $F(1,57)=.31, p=.58$ were significant when the target stimulus was presented on the left, although the pattern of results was the same.

For angry faces, there was no significant between subjects effect of group or within subjects effect of intensity when the target stimulus was presented on either side; however, there was a significant interaction between intensity and group (SP vs. control) when the target was presented on the right, $F(1$, $76)=4.77, p=.03 ; r=.24$, see Figure 3 , reflecting a pattern of significantly increased looking to high vs. low intensity faces amongst the offspring of mothers with social phobia, $t(31)=2.35, p=.02$, and a nonsignificant reduction in looking to high vs. low intensity angry faces among offspring of control mothers, $t(45)=.52, p=.60)$. As with fearful faces, this reflected a greater difference in looking times amongst infants of mothers with social phobia, but this time in the reverse direction to the pattern with fear faces (looking to high-low angry faces: SP mean $=0.23(.23)$, control mean $=-0.04(.53), t(76)=2.18, p=$. 03 ). This interaction was not significant when the target was presented on the left, $F(1,76)=2.55, p=$ $.12, r=.18)$, although the pattern of results was the same.

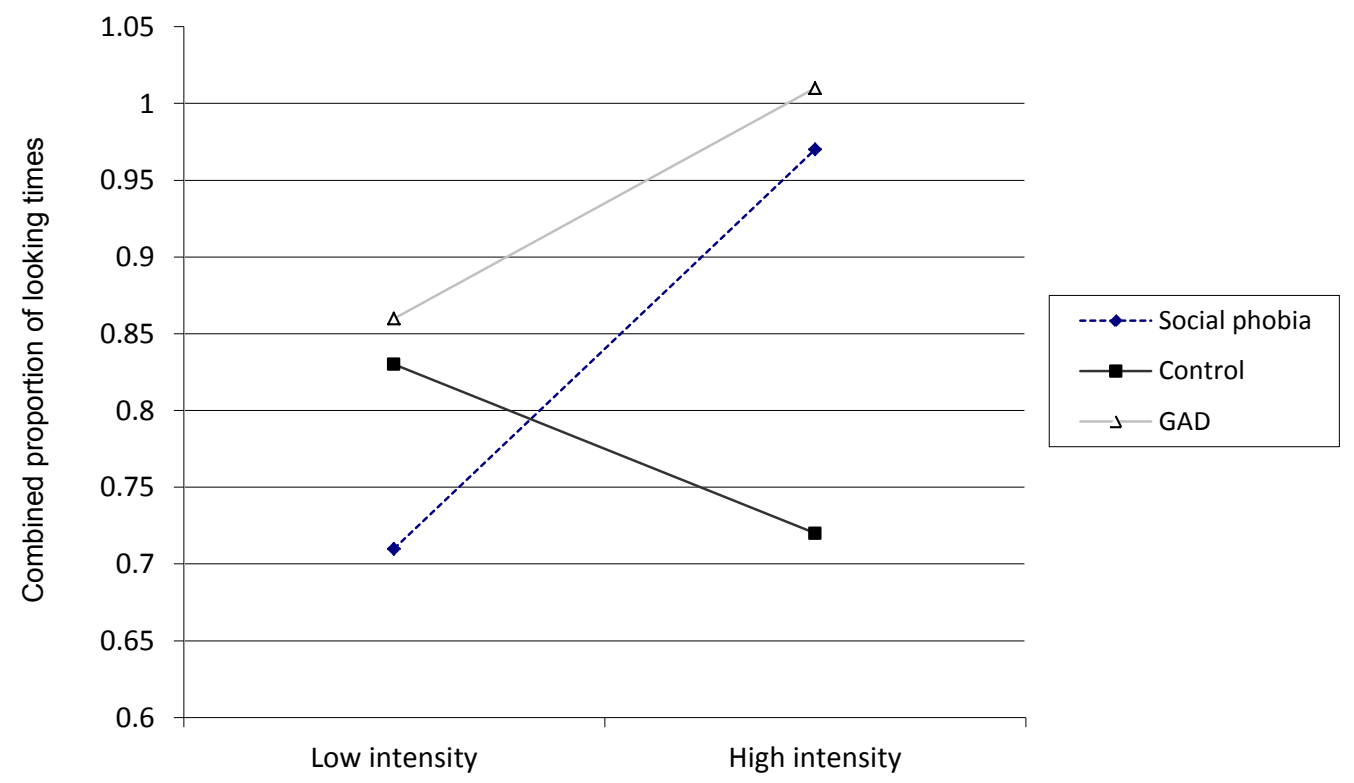

Figure 3: Interaction between participant group and level of emotional intensity of angry expressions (presented on the right side)

In order to address patterns of looking in greater detail (specifically to ascertain whether findings were consistent with initial hypervigilance/ avoidance), we compared infant responses to high intensity angry 
and fearful faces presented on the right, on the following: (i) which stimulus the infant looked to first (target/control), (ii) the length of the first look to target/control stimuli; and (iii) the total number of looks to target/control stimuli. For fearful faces, there was no significant difference between groups on where the infant looked first, $\chi^{2}(1)=51, p=.48$, or interactions between group and either the length of the first look to the target versus control stimuli, $F(1,75)=.82, p=.37$ ), or the number of looks to target vs. control stimulus, $F(1,74)=.03, p=.86$ ). For angry faces, there was a significant difference between infants of mothers with social phobia and control mothers in terms of where they looked first when presented with a high intensity face as part of the face pair, with infants of mothers with social phobia being less likely to look to the high intensity face first $\left(64 \%\right.$ vs. $\left.94 \% ; \chi^{2}(1)=9.25, p<.01\right)$. There were no significant interactions, however, between maternal group and either the length of the first look to the high intensity target versus control stimuli, $F(1,65)=.01, p=.91)$, or the number of looks to high intensity target vs. control stimulus, $F(1,65)=.01, p=.91)$.

\section{Specificity of the findings in comparison to infants of mothers with GAD}

360

361

362

363

364

365

366

367

368

369

370

371

372

373

374

375

376

377

378

379

380

381

382

383

384

385

386

387

388

389

390

391

Next, we were interested to determine to what extent the infant responses to emotional faces that discriminated infants in the social phobia and control groups, were specific to offspring of mothers with social phobia in comparison to offspring of GAD mothers. As above, the variables of interest were (i) the difference in looking time proportions to high and low intensity faces (proportion of time looking to high intensity - proportion of time looking to low intensity faces), and (ii) location of first looks (target vs. control); and for fearful faces (iii) the difference in looking time proportions to high and low intensity faces (proportion of time looking to high intensity - proportion of time looking to low intensity faces); all of which when presented on the right side.

When all three groups were considered, the main effects of intensity, the group and the group-intensity interaction were no longer significant for fear faces, however, the pattern of results suggest that the GAD group showed a less marked reduction in looking to high intensity fear faces than the social phobia group, and they appear to more closely resemble the pattern of the control group (see Figure 2).

For angry faces, the between subjects effect of group was not significant, $F(2,115)=1.91, p=.15$, but there was a significant effect of intensity, representing higher overall looking times to high intensity angry faces, $F(1,115)=4.65, p=.03, r=.20)$. The group $\times$ intensity interaction approached significance, $F(2$, $115)=2.49, p=.09, r=.13$, however this appeared to reflect the significant interaction when the social phobia and control groups were considered, as the interaction was not significant when analysed with either the social phobia, $F(1,70)=.41, p=.53$, or the control groups, $F(1,84)=2.38, p=.13, r=.17$. As shown in Figure 3, both infants of mothers with GAD and those of mothers with social phobia showed increased looking to high intensity angry faces, whereas infants of control mothers looked slightly less to high intensity than low intensity faces. Post-hoc analyses confirmed that the interaction was significant when only the social phobia and control groups were included, but not with the social phobia and GAD groups, $F(1,70)=.41, p=.53$, or the GAD and control groups, $F(1,84)=2.38, p=.13, r=.17$. As shown in Figure 3, the group difference appeared to be most marked for high intensity angry faces; therefore, follow-up analyses were conducted to explore this further. There was a significant group effect for the proportion of time spent looking at the high intensity angry faces presented on the right, $F(2,118)$ $=3.41, p=.04, r=.17$. Post-hoc t-tests confirmed that this reflected increased looking to high intensity angry faces by both the social phobia group, $t(79)=2.05, p=.04, r=.22$ and GAD group, $t(84)=2.51, p$ $=.01, r=.26$, in comparison to the control group, and no significance difference between the social phobia and GAD groups, $t(73)=.26, p=.80$.

A significant difference was found for the location of the first look compared across groups, $\chi^{2}(2)=9.49$, $p=.01$. This represented an increased rate of looking first to the high intensity angry face (vs. the 
control) for infants of non-anxious (94\%) followed by GAD (83\%) and finally socially phobic $(64 \%)$ mothers. Post-hoc analyses showed that this represented significant difference between the social phobia and control groups $\left(\chi^{2}(1)=9.25, p<.01\right)$, and a trend difference between the GAD and social phobia groups, $\chi^{2}(1)=2.97, p=.09$, but no significant difference between the GAD and control groups $\chi^{2}(1)=2.25, p=.13$.

\section{Associations with outcomes at 2 years}

To explore associations between looking responses to emotional faces at 10 weeks and outcomes at 2 years, we focused on those variables that had been identified as distinguishing between infant groups, i.e. for fearful faces, (i) the difference between looking times to high and low intensity faces; and for angry faces, (ii) the difference between looking times to high and low intensity faces, (iii) looking times to high intensity faces, and (iv) location of first looks (target vs. control) when presented on the right side. As few children scored above borderline/clinical cut-offs (i.e. anxiety problems $4.3 \%$; withdrawn $1.2 \%$; sleep problems $4.3 \%$ ), CBCL variable scores were dichotomised on the basis of frequency distributions (which led to data being split at the median in all cases).

Logistic regression analyses were conducted, with dichotomised $\mathrm{CBCL}$ scores as the dependent variables, and group, looking response (i.e. the four looking response variables outlined above) and their interaction as the predictor variables. For $\mathrm{CBCL}$ anxiety problems, none of the main effects of group or looking responses were significant, however, there was a significant effect of the interaction between (social phobia) group and difference in looking time to high and low intensity fear faces, $\exp (B)=14.28$, $p=.04$, see Table 3. To probe the interaction, the binary logistic regression was run to examine the effects of group when the difference in looking to high and low intensity fear faces was set (at the mean plus one standard deviation above and one standard deviation below the mean). This confirmed a significant effect of social phobia group when the difference score was high, $\exp (B)=7.39, p=.05$. The effect of GAD group when the difference score was high approached significance, $\exp (B)=4.68, p=.06$. Group effects were not significant when the difference score was low.

Table 3: Prediction of CBCL anxiety problems at two years from maternal group and high-low looking to fear faces

\begin{tabular}{|c|c|c|c|c|}
\hline & \multirow[b]{2}{*}{$\mathrm{B}(S E)$} & \multicolumn{3}{|c|}{$95 \% \mathrm{Cl}$ for Odds Ratio } \\
\hline & & Lower & Odds ratio & Upper \\
\hline \multicolumn{5}{|l|}{ Included } \\
\hline Maternal group (SP vs. others) & $.69(.63)$ & .58 & 1.19 & 6.90 \\
\hline Maternal group (GAD vs. others ) & $.62(.58)$ & .60 & 1.16 & 5.75 \\
\hline Looking to high fear/ Looking to low fear & $0.92(.76)$ & .09 & 1.46 & 1.77 \\
\hline Group (SP) x Looking response & $2.66(1.34)^{*}$ & 1.03 & 3.93 & 197.64 \\
\hline Group (GAD) x Looking response & $-.57(.40) \dagger$ & .87 & 2.01 & 48.65 \\
\hline
\end{tabular}

$R^{2}=.09$ (Cox a\& Snell), $R^{2}=.12$ (Nagelkerke), Model $\chi^{2}(5)=7.48, p=.19$

As looking responses did not have an independent effect on $\mathrm{CBCL}$ anxiety problems, mediation hypotheses were not pursued further. There were no significant main effects or interactions in relation to sleep or withdrawn problems. 


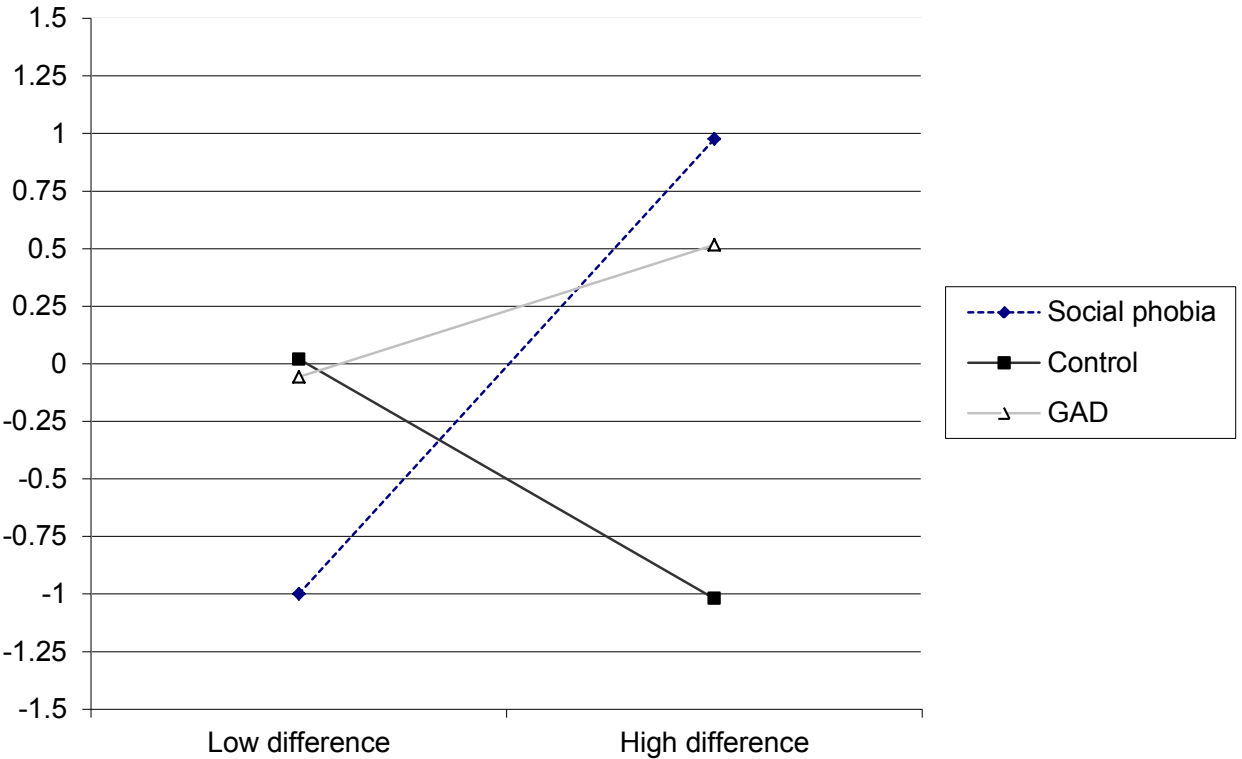

Looking to high intensity fearful face minus Looking to low intensity fearful face

Figure 4: Prediction of CBCL anxiety at two years from maternal group and difference between high and low looking to fear faces

\section{Discussion}

Socially anxious adults and children show biases in their looking responses to emotional faces (e.g. Bögels \& Mansell, 2004; Garner, 2010), and consistent patterns have been found among infants at hereditary risk of social phobia (Creswell et al., 2008). This study set out to evaluate whether these patterns are specific to infants at risk of social phobia, in comparison to infants at more general risk of anxiety problems (i.e. infants of mothers with GAD), and to identify whether early responses to emotional expressions are associated with emerging anxiety symptoms assessed at two years of age.

As the current study limited the index group of participants to infants of mothers with pure social phobia (i.e. not those with comorbid GAD), we first set out to establish whether the findings from our previous report were maintained with this restricted group (Creswell et al., 2008). The results were partially replicated. As before, there were no group differences in overall looking times to particular stimuli, however groups did differ in their relative looking to high and low intensity stimuli (each presented alongside a $50 \%$ intensity expression), when target stimuli were presented on the right side. As before, we found a significant interaction between maternal group and emotional intensity for fear faces, reflecting less relative looking time to high intensity faces among infants of mothers with social phobia compared to non-anxious mothers. In other words, infants of socially phobic mothers preferred the $50 \%$ control expression to the high intensity stimulus, but preferred the low intensity face to the $50 \%$ control expression; thus, infants of mothers with social phobia showed a preference in all cases for the lower intensity fear expression of the pair. For infants of control mothers, however, there was no significant evidence for a preference. In our previous report we noted that, for fearful faces, infants of socially phobic mothers looked less frequently to the target stimulus first, however, this was not significant with 
this smaller group of infants of mothers with pure social phobia. The effect size was substantially lower than in our previous report, which suggests that the effect may have been substantially influenced by the inclusion of comorbid cases previously, rather than the difference arising as a function of sample size.

In our previous paper we did not find any significant results in relation to angry faces; however, with the current pure social phobia group, we did find a significant interaction between group and emotional intensity (again when the target as presented on the right side). For angry faces, we saw the reverse pattern from fear faces, i.e. infants of socially phobic mothers looked relatively more to high intensity faces, whereas infants of non-anxious mothers looked relatively more to low intensity faces. In other words, infants of socially phobic mothers showed a preference for the higher intensity face in each face pair (when presented with a high intensity face next to a $50 \%$ face they would show more looking to the high intensity face, and when presented with a low intensity face next to a $50 \%$ face they would show more looking to the $50 \%$ face). The infants of the non-anxious mothers showed the reverse pattern. In terms of initial looking, the index infants were more likely to look first to the $50 \%$ expression than the control group, but there were no differences in the length of the first look or number of looks overall to each stimulus. Overall, the findings for neither fear nor angry faces are consistent with a pattern of hypervigilance-avoidance for threat, however, overall, infants of mothers with social phobia appear to attend preferentially away from higher intensity fear faces, and towards higher intensity angry faces.

When infants of mothers with GAD were also considered, the interaction between group and emotional intensity for fear faces was no longer significant, which most probably reflected the loss of power from the inclusion of a third group. For angry faces, however, the social phobia and GAD groups displayed a similar pattern of responding, in which both looked more to high intensity angry faces, in comparison to the control infants. When high intensity faces were presented alongside the $50 \%$ control, infants of nonanxious mothers were significantly more likely to look to the target first in comparison to the social phobia and GAD groups, suggesting that the increased overall looking to high intensity faces seen amongst the anxiety groups was not based on early hypervigilance to the high intensity face.

The pattern of results is consistent with the conclusion that risk for social anxiety may be associated with avoidance of fearful faces; whereas risk for anxiety generally is associated with a vigilance towards angry faces. This conclusion is consistent with previous findings in a community population that indicated that social anxiety symptoms are associated with reduced attention to fearful faces (Stirling et al., 2006), and findings from clinical populations that children with GAD show attention towards angry faces (Monk et al., 2008; Waters et al., 2008). The results are contrary, however, to findings that reduced attention to angry faces was associated with levels of social anxiety in community participants (Stirling et al., 2006) and also the presence of a GAD diagnosis (Monk et al., 2006). While our results require replication, it does seem plausible that, in infants, anger and fear presentations may evoke different response patterns, as these expressions serve clearly different functions, with angry faces reflecting a threat, and fear faces reflecting a warning sign. According to this interpretation, our results would suggest that a hereditary risk for anxiety is associated with a bias towards threat information; but a hereditary risk for social anxiety specifically is associated with a bias away from warning signs.

In terms of associations with children's anxiety-related symptoms at two years of age, we considered symptoms of social-withdrawal, general anxiety, and also sleep problems. Findings were strongest in relation to general anxiety problems. Specifically, risk for problems was increased if infants of mothers with social phobia displayed higher relative looking times to high (vs. low) intensity fear faces at 10 weeks. In other words, when infants of socially anxious mothers showed a preference for low intensity fear faces this was associated with a better outcome at two years, than when they showed a preference for high intensity fear faces. We did not find any significant main or interaction effects in relation to sleep 
problems at two years. Whilst conclusions must remain tentative, this may reflect these particular problems being more a response to environmental factors (e.g. care practices) than endogenous factors.

The interactive effects of infant looking response and maternal group are intriguing. While infants of mothers with social phobia are more likely to show reduced looking to fearful faces, those that do not show this pattern appear to be at increased risk of anxiety symptoms at two years of age. Any conclusions must remain tentative and the results require replication, however one possible explanation is that a tendency for infants to prefer fear faces may elicit particular responses from anxious (but not non-anxious) mothers. For example, anxious mothers may respond to infant interest in fear faces, and related stimuli, with reduced levels of social encouragement, which may lead to increases in infant anxiety as the child develops social understanding through social referencing processes in the period between our two assessment points (e.g. Murray et al., 2008). Future studies would benefit from the inclusion of measures to test and power to test these transactional processes.

It is important to note that, for infant responses to emotional expressions, the side of presentation of the index face was important and the interaction effects of group and intensity of fearful and angry expressions were most pronounced for presentations on the right. Our results are consistent with those of other studies with adults (Mogg \& Bradley, 1999, 2002), suggesting that lateralised brain mechanisms may play a critical role in the processing of face stimuli (Morris, Ohman, \& Dolan, 1998). Conclusions with regards the specificity of findings to stimuli presented on the right must, however, remain tentative.

There are various reasons to consider our results with caution. In particular, significant results reflect modest effects sizes and, as such, maternal group appears to explain a relatively small proportion of the variance in children's responses to emotional expressions. Due to the novelty of the approach, we did not want to be overly conservative in an attempt to reduce Type 1 error, however, it must be acknowledged that a relatively large number of univariate tests were conducted. As young infants can be expected to frequently become fatigued or unsettled, the number of trials that we administered was low (e.g. two high intensity faces of each emotion type on each side of presentation). Even so, there were a substantial number of infants with incomplete trials, which limited the use of multivariate analyses and restricted the power of the study, which may have led to Type 2 error. Second, our assessment of child adjustment was based on maternal report. While the $C B C L$ is a widely used measure with established reliability, maternal reports may be influenced by levels of maternal anxiety (Briggs-Gowan, Carter, \& Schwab-Stoar, 1996). In addition, it is important to note that while differences were found in relation to CBCL outcomes, very few children scored within the borderline or abnormal ranges, and as such differences reflected relatively small differences in nonclinical symptom levels. Finally, the current study is not able to differentiate between different classes of risk, such as genetic risk, effects of antenatal anxiety and stress (e.g. O'Connor, Heron, Golding, \& Glover, 2003; ven den Bergh \& Marcoen, 2004) or antenatal medication.

With these limitations in mind, the current study provides further support for the integral bias hypothesis, in that evidence for biases in responses to emotional expressions was evident in young infants at hereditary risk of anxiety and social phobia (e.g. Richards et al., 2002). Whereas attention towards angry faces is associated with a general risk for anxiety, attention away from high intensity fear faces is associated with a more specific risk for social anxiety. Infants of anxious mothers who fail to show this early pattern, however, appear to be at increased risk of anxiety symptoms at two years. Aside from the need for replication, future studies would benefit from longitudinal assessments of continuity and change in responses to emotional expressions in order to track their associations with anxiety symptoms in later childhood and adolescence. 


\section{Acknowledgements}

This work was supported by a programme grant from the Medical Research Council (UK) to Lynne Murray and Peter Cooper (ref G9324094). Cathy Creswell is supported by an MRC Clinician Scientist Fellowship (ref G0601874). We are grateful to Susan Campbell, Amber Davis, Melanie Edwards, Rachel Kelly, Paula Liberton, Chiara Navarra, Monika Parkinson, Joanna Pearson, Melanie Royal-Lawson, Caroline Sack, Barbara Sana, Hannah Seabrook, and Sheila Summers for assistance, variously, with recruitment and assessment of study participants, and coding of interaction data. We thank Nancy Snidman and Jerome Kagan for their support with assessment of infant behavioural inhibition. We thank Andy Young, Mark Johnson, John Morton and Francesca Simion for their invaluable advice on the design of this study.

\section{References}

Achenbach, T.M. \& Rescorla, L. (2000). Manual for the AEBA preschool forms \& profiles: An integrated system of multi-informant assessment. ASEBA, Burlington, VT.

Andrews, G. (1996). Comorbidity in neurotic disorders: The similarities are more important than the differences. In R. M. Rapee (Ed.), Current controversies in the anxiety disorders. New York: Guilford Press. pp. 3-20.

Beatty, M. J., Heisel, A. D., Hall, A. E., Levine, T. R., \& La France, B. H. (2002). What can we learn from the study of twins about genetic and environmental influences on interpersonal affiliation, aggressiveness, and social anxiety?: A meta-analytic study. Communication Monographs, 69(1), 118.

Bienvenu, O.J., Hettema, J.M., Neale, M.C.,Prescott, C.A., \& Kendlar, K.S. (2007). Low extraversion and high neuroticism as indices of genetic and environmental risk for social phobia, agoraphobia and animal phobia. American Journal of Psychiatry, 164, 1714-1721.

Bögels, S.M. \& Mansell, W. (2004). Attention processes in the maintenance and treatment of social phobia: Hypervigilance, avoidance, and self-focussed attention. Clinical Psychology Review, 24, $827-$ 856.

Bradley, B.P., Mogg, K., \& Lee, S.C. (1997). Attentional bias for negative information in induced and naturally occurring dysphoria. Behaviour Research and Therapy, 35, 911-927.

Bradley, B.P., Mogg, K.,falla, S.J., \& Hamilton, L.R. (1998). Attentional bias for threatening facial expressions in anxiety: manipulation of stimulus duration. Cognition and Emotion, 12, 737-753.

Bradley,B.M., Mogg, K., White, J., Groom, C., \& de Bono, J. (1999). Attentional bias for emotional faces in generalized anxiety disorder. British Journal of Clinical Psychology, 38, 267-278.

Briggs-Gowan, M.J., Carter, A.S., \& Schwab-Stone, M. (1996). Discrepancies among mother, child and teacher reports: Examining the contributions of maternal depression and anxiety. Journal of Abnormal Child Psychology, 24, 749-765.

Creswell, C., Woolgar, M., Cooper, P., Giannakakis, A., Schofield, E., Young, A.W., \& Murray, L. (2008). Processing of faces and emotional expressions in infants at risk of social phobia. Cognition and Emotion, 22, 437-458.

Ekman, P. and Friesen, W.V. (1976). Pictures of facial affect. Palo Alto, California: Consulting Psychologists Press.

Eley, T. C. (1999). Behavioral genetics as a tool for developmental psychology: Anxiety and depression in children and adolescents. Clinical Child and Family Psychology Review, 2(1), 21-36.

Elizabeth, J., King, N., Ollendick, T.H., Gullone, E., Tonge, B., Watson, S. et al (2006). Social anxiety disorder in children and youth: a research update on aetiological factors. Counselling Psychology Quarterly, 19, 151-163.

Comment [CE9]: When referencing a paper with more than 7 authors please reference the first 6 authors followed by . and then the last author. If there are 7 or less authors then please include all the authors in the reference. 
First, M.B., Spitzer, R.L., Gibbon, M., et al (1995). Structured Clinical Interview for DSM-IV Axis I Diagnoses. New York: Biometrics Research Department.

Furmark, T. (2002). Social phobia: Overview of community surveys. Acta Psychiatrica Scandinavica, 105, 84-93.

Garner, M., Mogg, K., \& Bradley, B.P. (2006). Orienting and maintenance of gaze to facial expressions in social anxiety. Journal of Abnormal Psychology, 115, 760-770.

Garner, M. (2010). Selective attention to threat in childhood anxiety: Evidence from visual probe paradigms. In J.A. Hadwin \& A.P. Field (Eds) Information processing biases and anxiety: A developmental perspective. West Sussex: Wiley Blackwell; pp 77-108.

Gregory, A.M. \& O'Connor, T.G. (2002). Sleep problems in childhood: a longitudinal study of developmental change and association with behavioural problems. Journal of the American Academy of Child and Adolescent Psychiatry, 41, 964-971.

Gregory, A.M., Van der Ende, J., Willis, T.A., \& Verhulst, F.C. (2008). Parent reported sleep problems during development and self-reported anxiety/depression, attention problems and aggressive behaviour later in life. Archives of Pediatric and Adolescent Medicine, 162, 330-335.

Johnson, E.O., Chilcoat, H.D., \& Breslau, N. (2000). Trouble sleeping and anxiety/depression in childhood. Psychiatry Research, 94, 63-102.

Joormann, J. \& Gotlib, I.H. (2006). Is this happiness I see? Biases in the identification of emotional faces in depression and social phobia. Journal of Abnormal Psychology, 115, 705-14.

Joormann, J., Talbot, L., \& Gotlib, I.H. (2007). Biased processing of emotional information in girls at risk for depression. Journal of Abnormal Psychology, 116, 135-143.

Kendler, K. S., Neale, M. C., Kessler, R. C., Heath, A. C., et al. (1992). The genetic epidemiology of phobias in women: The interrelationship of agoraphobia, social phobia, situational phobia, and simple phobia. Archives of General Psychiatry, 49(4), 273-281.

Kendler, K. S., Myers, J., Prescott, C. A., \& Neale, M. C. (2001). The genetic epidemiology of irrational fears and phobias in men. Archives of General Psychiatry, 58(3), 257-265.

Kindt, M. \& van den Hout, M. (2001). Selective attention and anxiety: A perspective on developmental issues and the causal status. Journal of Psychopathology and Behavioural Assessment, 23, 193-202.

Lieb, R., Wittchen, H.U., Hofler, M., Fuetsch, M., Stein, M.B., \& Merikangas, K.R. (2000). Parental psychopathology, parenting styles, and the risk of social phobia in offspring: A prospectivelongitudinal community study. Archives of General Psychiatry, 57, 859-866.

Lochner, C., Hemmings, S., Seedat, S., Kinnear, C., Schoeman, R., Annerbrink, K., et al. (2007). Genetics and personality traits in patients with social anxiety disorder: a case-control study in South Africa. Europeam Neuropsychopharmacology, 17, 321-327.

Mancini, C., van Ameringen, M., Szatmari, P., Fugere, C., \& Boyle, M. (1996). A high-risk pilot study of the children and adults with social phobia. Journal of the American Academy of Child and Adolescent Psychiatry, 35, 1511-1517.

Mansell, W., Clark, D.M., Ehlers, A., \& Chen, Y.P. (1999). Social anxiety and attention away from emotional faces. Cognition and Emotion, 13, 673-690.

McClure, E.B. \& Nowicki, S. (2001). Associations between social anxiety and nonverbal processing skills in preadolescent boys and girls. Journal of Nonverbal Behavior, 25, 3-19.

Mogg, K. \& Bradley, B.P.(1998). A cognitive-motivational analysis of anxiety. Behaviour Research and Therapy, 36, 809-848.

Mogg, K. \& Bradley, B. (2002). Selective orienting of attention to masked threat faces in social anxiety. Behaviour Research and Therapy, 40, 1403-1414.

Mogg, K., Bradley, B.P., Miles, F., \& Dixon, R. (2004). Time course of attentional bias for threat scenes: testing the vigilance-avoidance hypothesis. Cognition and Emotion, 18, 689-700. 
Mogg, K., Bradley, B., de Bono, J., \& Painter, M. (1997). Time course of attentional bias for threat information in non-clinical anxiety. Behaviour Research and Therapy, 37, 595-604.

Monk, C.S., Nelson, E.E., McClure, E.B., Mogg, K. et al.(2006). Ventrolateral prefrontal cortex activation and attentional bias in response to angry faces in adolescents with generalized anxiety disorder. American Journal of Psychiatry, 163, 1091-1097.

Monk, C.S., Telzer, E.H., Mogg, K., Bradley, B., Mai, X. et al. (2008). Amygdala and ventrolateral prefrontal cortex activation to masked angry faces in children and adolescents with generalized anxiety disorder. Archives of General Psychiatry, 65, 568-576.

Murray, L., Cooper, P., Creswell, C., Schofield, E., \& Sack, C. (2007). The effects of maternal social phobia on mother-infant interactions and infant social responsiveness. Journal of Child Psychology and Psychiatry, 48, 45-52.

Murray, L., DeRosnay, M., Pearson, J. Bergeron, C., Schofield, L., Royal-Lawson, M. \& Cooper, P.J. (2008). Intergenerational transmission of maternal social anxiety: the role of the social referencing process. Child Development, 79, 1049-1064.

O'Connor, T.G., Heron, J., Golding, J., Glover, V. \& the ALSPAC Study Team (2003). Maternal antenatal anxiety and behavioural/emotional problems in children: a test of a programming hypothesis. Journal of Child Psychology and Psychiatry, 44, 1025-1036.

Pascalis, O., de Schonen, S., Morton, J., Deruelle, C., \& Fabre-Grenet, M. (1995). Mother's face recognition by neonates: a replication and an extension. Infant Behaviour and Development, 18, 7985.

Richards, A., French, C., Nash, G, Hadwin, J.A., \& Donnelly, N. A. (2007). Comparison of selective attention and facial processing biases in typically developing children high and low in self-reported trait anxiety. Development and Psychopathology, 18, 481-495.

Rosenbaum, J., Biederman, J., Bolduc, E., Hirschfeld, D., Faraone, S., \& Kagan, J. (1992). Comorbidity of parental anxiety disorders as risk for childhood onset anxiety in inhibited children, American Journal Psychiatry, 149, 475-581.

Roy, A.K., Vasa, R.A., Bruck, M., Mogg, K., Bradley, K.et al. (2008). Attention bias toward threat in pediatric anxiety disorders. Journal of the American Academy of Child and Adolescent Psychiatry, 47, 1189-1196.

Stirling, L.J., Eley, T.C., \& Clark, D.M. (2006). Preliminary evidence for an association between social anxiety symptoms and avoidance of negative faces in school-age children. Journal of Clinical Child and Adolescent Psychology, 35, 440-445.

Stoleru, S., Nottelmann, E.D., Belmont, B., \& Ronsaville (1997). Sleep problems in children of affectively ill mothers. Journal of Child Psychology and Psychiatry, 38, 831-841.

Van den Bergh, B.R.H. \& Marcoen, A. (2004). High antenatal maternal anxiety is related to ADHD symptoms, externalising problems, and anxiety in 8 and 9 year olds. Child Development, 75, 10851097.

Waters, A.M., Mogg, K., Bradley, B.P., \& Pine, D.S. (2008). SAttentional bias for emotional faces in children with generalized anxiety disorder. Journal of the American Academy of Child and Adolescent Psychiatry, 47, 435-442.

Yoon, K.L., \& Zinbarg, R.E. (2007). Threat is in the eye of the beholder: Social anxiety and the interpretation of ambiguous facial expressions. Behaviour Research and Therapy, 45, 839-847.

Young, A.W., Perrett, D.I., Calder, A.J., Sprengelmeyer, R. and Ekman, P. (2002). Facial expressions of emotion: stimuli and tests (FEEST). Bury St. Edmunds: Thames Valley Test Company.
Comment [CE12]: When referencing a paper with more than 7 authors please reference the first 6 authors followed by ... . and then the last author. If there are 7 or less authors then please include all the authors in the reference.

Comment [CE13]: When referencing

a paper with more than 7 authors

please reference the first 6 authors followed by .... and then the last author. If there are 7 or less authors then please include all the authors in the reference.

Comment [CE14]: When referencing a paper with more than 7 authors please reference the first 6 authors followed by ... and then the last author. If there are 7 or less authors then please include all the authors in the reference. 